REVIEW

\title{
Unnatural selection: reducing antibiotic resistance in neonatal units
}

\section{Isaacs}

Arch Dis Child Fetal Neonatal Ed 2006;91:F72-F74. doi: 10.1136/adc.2005.074963

Antibiotic use selects for antibiotic resistant bacteria. This is an example of rapid Darwinian natural selection in action. It occurs in neonatal intensive care units with the use of parenteral antibiotics, and in the community with oral antibiotic use. A 10 point plan is put forward to reduce antibiotic resistance in neonatal units.

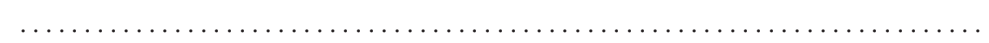

Correspondence to: Dr Isaacs, Department of Allergy, Immunology \& Infectious Diseases, Children's Hospital at Westmead, Westmead Sydney, 2122, NSW, Australia; davidi@chw. edu.au

Accepted 6 June 2005
A ntibiotic use selects for antibiotic resistant bacteria. $^{1-3}$ This is an example of rapid Darwinian natural selection in action. It has been shown to occur in neonatal intensive care units with the use of parenteral antibiotics, ${ }^{1}$ and in the community with oral antibiotic use. ${ }^{2} 3$ When penicillin was first used in the 1940s and 1950s, Staphylococcus aureus was always exquisitely sensitive to benzyl penicillin. Within a very short period of time, most disease-causing strains of $S$ aureus were penicillin resistant. The antibiotic pressure exerted by widespread penicillin use had selected naturally occurring mutant strains of $S$ aureus that were inherently resistant to penicillin.

Broad spectrum antibiotics might be expected to be more potent selectors of antibiotic resistant bacteria than narrow spectrum antibiotics, and this has indeed proved to be the case in clinical practice. $^{1-4}$ The selection of multiresistant bacteria is causing major problems in neonatal intensive care units. Ampicillin and third generation cephalosporins select for Gram negative bacilli that produce extended spectrum $\beta$ lactamases, which render the bacterium resistant to many antibiotics, not just $\beta$ lactams. ${ }^{15-11}$ The carbapenems, such as imipenem and meropenem, are used in the laboratory to induce organisms carrying repressed $\beta$ lactamase genes to express these genes and produce $\beta$ lactamases. ${ }^{12}$ Therefore extensive use of imipenem and meropenem will select for $\beta$ lactamaseproducing organisms, as well as for organisms resistant to imipenem. ${ }^{13}$ Gram positive bacteria can carry genes conferring vancomycin resistance, such as vancomycin resistant enterococci, and genes coding for methicillin resistance, such as methicillin-resistant Staphylococcus aureus (MRSA) and methicillin-resistant Staphylococcus epidermidis. ${ }^{5}$ Prolonged use of broad spectrum antibiotics is also causing a rising incidence of severe fungal sepsis, even in full term babies, in countries such as India. ${ }^{14}$

Antibiotic use selects for antibiotic resistant bacteria, but we still need to use antibiotics to treat babies. The real problem is that prolonged antibiotic use, particularly with broad spectrum antibiotics, is highly likely to select for highly resistant bacteria. Such excessive use of broad spectrum antibiotics for prolonged periods might be seen as "unnatural selection".

\section{INDUSTRIALISED AND DEVELOPING COUNTRIES}

For years neonatologists in industrialised countries in North America, Europe, and Australia have been reporting problems with multiresistant bacteria such MRSA and Gram negative bacilli that produce extended spectrum $\beta$ lactamase. ${ }^{14-9}$ Similar reports are now appearing from developing countries. ${ }^{1013} 14$ Two recent reviews of neonatal infections in developing countries have highlighted alarming rates of antibiotic resistance. $^{1516}$

We recently surveyed 19 Asian neonatologists, whose names are at the end of this paper, from Malaysia (six), China (four), the Philippines (two), Sri Lanka (two), Thailand (two), and one each from India, Macau, and Mongolia. Seventeen (89\%) reported significant problems with sepsis caused by multiresistant Gram negative bacilli, and $16(84 \%)$ reported sepsis due to MRSA. Fifteen (79\%) reported high rates of nosocomial sepsis with these organisms. Seven $(37 \%)$ said that they experienced delay in obtaining microbiology results, while only four $(21 \%)$ felt unable to rely on the validity of cultures. Nevertheless, there is a strong tendency, at least in India, to start antibiotics without taking blood cultures first, and/or to continue antibiotics if the baby is "sick", even if blood cultures are negative. ${ }^{14}$

\section{NEW ANTIBIOTICS}

Pharmaceutical companies are understandably keen to promote their antibiotics as "broad spectrum", so that the antibiotics will be used in a wide range of clinical situations where the causative organism is unknown. This is of course a common situation when treating neonates, because the rapidity of infection generally necessitates starting empiric antibiotic therapy without knowing the infecting organism. Being broad spectrum is a double edged sword for an antibiotic. Short term use provides cover against a wide range of organisms, but long term use is a powerful selector for resistance. Imipenem, for example, has activity against a wide range of Gram negative bacilli and Gram positive cocci (except for MRSA), but extensive use of imipenem selects for highly resistant bacteria. ${ }^{13}$

When we have used antibiotics irresponsibly, and babies in our neonatal units are extensively 
colonised with highly resistant organisms, we may turn to the major pharmaceutical companies and ask why they are not producing new antibiotics. Of course, if a new antibiotic is developed, we do not want organisms to become resistant to it rapidly, so we restrict its use, as severely as we can. It is not surprising that the price of new antibiotics is exorbitant, if we want an antibiotic that will treat the highly resistant organisms we have selected, but then we want to use the new antibiotic as sparingly as possible.

It is unrealistic to expect pharmaceutical companies to solve the problems we have created by excessive and irresponsible use of antibiotics.

\section{RESTRICTING ANTIBIOTIC USE}

Which are the best antibiotics to use? It is a fallacy to think that broad spectrum antibiotics are better because they cover more organisms. On the contrary, for that very reason they are more potent at selecting for resistant organisms. In a hugely important study in neonatal units in the Netherlands, de Man et al $^{1}$ showed that empiric therapy using the "narrow spectrum" antibiotics, penicillin and tobramycin, was significantly less likely to select for resistant organisms than using "broad spectrum" amoxycillin and cefotaxime.

Almost all experts agree that the best empiric regimen for neonates is a penicillin or semisynthetic penicillin together with an aminoglycoside. ${ }^{17-21}$ The choice of the penicillin will depend on the organisms causing sepsis. If it is necessary to cover for staphylococci, then oxacillin, cloxacillin, or flucloxacillin may be most appropriate. ${ }^{17}$ In developing countries, it may be necessary to cover for staphylococci from birth, whereas staphylococcal infections are rare in industrialised countries before the third day of age. ${ }^{21}{ }^{22}$ Empiric vancomycin is not necessary unless MRSA is common, ${ }^{17-21}$ and it is important to restrict vancomycin use in all countries because of the risk of selecting for vancomycin resistant organisms. The choice of aminoglycoside also depends on local data. If the colonising and infecting bacteria in a neonatal unit all become resistant to gentamicin, for example, they may become sensitive again after a prolonged period using an aminoglycoside to which they are sensitive-for example, netilmicin. ${ }^{22}$

In some countries, the organisms colonising babies from birth have high rates of antibiotic resistance, suggesting that community antibiotic use has resulted in the mothers being colonised with resistant organisms. ${ }^{14}$ The antibiotics of choice for early onset neonatal sepsis will then need to be tailored to cover the likely organisms-for example, it may be necessary to use empiric flucloxacillin and netilmicin for suspected early sepsis if there are high rates of early onset infection with $S$ aureus and with gentamicin resistant Gram negative bacilli.

Using antibiotics in rotation has been effective in some settings in reducing resistance. ${ }^{23}{ }^{24}$ However, most developed countries now place restrictions on which antibiotics may be used and when, and such restrictions have been shown to reduce antibiotic resistance. In a study from Brazil, neonatal unit healthcare associated infections were reduced from $32 \%$ to $11 \%$ after education and restriction of the use of cephalosporins. ${ }^{25}$

How does one change antibiotics, if most of the babies on a neonatal unit are already colonised with one or more multiresistant organisms? One way would be to separate (or "cohort") the babies currently on the unit from any new admissions. The babies colonised with the resistant organisms-for example, MRSA-would be given the appropriate antibiotics (in this case vancomycin with gentamicin) if they developed suspected sepsis. The new admissions would be given flucloxacillin and gentamicin. Provided that the new admissions are protected, by hand washing and good
Ten point plan to reduce antibiotic resistance in neonatal units

(1) Always take cultures of blood (and perhaps cerebrospinal fluid and/or urine) before starting the infant on antibiotics.

(2) Use the narrowest spectrum antibiotics possible, almost always a penicillin-for example, benzylpenicillin, flucloxacillin, piperacillin, ticarcillin-and an aminoglycoside-for example, gentamicin, netilmicin, amikacin.

(3) Do not start treatment, as a general rule, with a third generation cephalosporin-for example, cefotaxime, ceftazidime-or a carbapenem - for example, imipenem, meropenem.

(4) Develop local and national antibiotic policies to restrict the use of expensive, broad spectrum antibiotics.

(5) Trust the microbiology laboratory: rely on the blood culture results.

(6) Do not believe that abnormal results for a non-specific test, such as a raised serum $C$ reactive protein, means that the baby is definitely septic.

(7) If blood cultures are negative at two to three days, it is almost always safe and appropriate to stop antibiotics.

(8) Try not to use antibiotics for long periods.

(9) Treat sepsis, but not colonisation.

(10) Do your best to prevent nosocomial infection, by reinforcing infection control, particularly hand washing.

hygiene, from the babies colonised with resistant organisms, it should be possible to introduce "narrow spectrum" antibiotics and continue their use once all babies colonised with resistant organisms are discharged.

\section{BLOOD CULTURE RESULTS AND DURATION OF ANTIBIOTICS}

It is important that microbiology laboratories use blood culture techniques that are known to be highly sensitive and reliable, such as the BacTec or BacT Alert systems. Once such systems are in place, doctors must rely on blood culture results. Because of the relatively high number of organisms in the bloodstream in neonatal septicaemia, blood cultures are extremely reliable. ${ }^{26}{ }^{27}$ In a Mexican study of infants, $2.2 \mathrm{ml}$ blood was taken, and divided into $2 \mathrm{ml}$ and $0.2 \mathrm{ml}$ aliquots. If the $2 \mathrm{ml}$ blood culture was positive, the $0.2 \mathrm{ml}$ sample was also positive in $95 \%$ of cases. ${ }^{28}$ It has been shown in industrialised countries that if blood cultures taken for suspected late onset sepsis are negative after 48-72 hours, antibiotics can in general be stopped, and babies do not relapse. ${ }^{29} 30$

If a baby with suspected sepsis is started on antibiotics, the antibiotics are not being used for prophylaxis but to treat possible sepsis. If cultures are negative two to three days later, antibiotics should almost always be stopped..$^{29} 30$ Exceptions for stopping antibiotics would be where the baby has early or late onset pneumonia, because the sensitivity of blood cultures is only about $50 \%{ }^{31}$ It may also be wise to continue antibiotics despite negative blood cultures in a baby with suspected early onset sepsis, if the baby is heavily colonised with Listeria or Group B Streptococcus and the mother did not receive intrapartum antibiotics, particularly if a lumbar puncture was not performed.

Prophylactic antibiotics are ineffective in preventing sepsis, whether the antibiotics are given because the baby is intubated, because the baby has an umbilical artery or catheter, a central line, or a chest drain. ${ }^{31}$ If bacteria are 
grown from an endotracheal tube culture, that is colonisation unless the baby has pneumonia. The baby should only be treated for pneumonia (on radiograph) or for sepsis, but not for colonisation. Treatment does not reduce colonisation (the endotracheal tube cultures will remain positive) and does not prevent sepsis, but is likely to increase antibiotic resistance. ${ }^{32}$

\section{PREVENTION OF NOSOCOMIAL INFECTION}

Although it is important that antibiotic use improves, prevention of infection should not be neglected. Simple, cheap but effective strategies are available. ${ }^{16}{ }^{33}{ }^{34}$ Improved hand washing has consistently been shown to reduce the incidence of nosocomial sepsis. We all know we should wash our hands, but we could all do better. We are worst at washing our hands when we are busiest. Although this seems logical, unfortunately it is when we are busiest that nosocomial infections are most likely. We should try to wash our hands more, not less, as we get busier. Early introduction of enteral feeds, preferably breast milk, to babies in intensive care allows cannulas to be removed more quickly, reducing the risk of sepsis. ${ }^{31}$

\section{ACKNOWLEDGEMENTS}

The following neonatologists kindly completed questionnaires on neonatal infection: Professor Nem-Yun Boo, Universiti Kebangssan, Kuala Lumpur, Malaysia; Dr Lee Gaik Chan, Kuching, Malaysia; Dr Jackie Ho, Ipoh General Hospital, Ipoh, Malaysia; Dr Jimmy Kok-Foo Lee, Hospital Kuala Terengganu, Malaysia; Professor Chin-Theam (CT) Lim, University of Malaya, Kuala Lumpur, Malaysia; Dr Hans Van Rostenberghe, Universiti Sains Malaysia, Kelantan, Malaysia; Professor Cuiqing Liu, Heibei Childreen's Hospital, Heibei, China Professor Bai Yao, PUMC Hospital, China; Professor Xin Xiao, Jinan University, Guangzhou, China; Dr Zhou Xiaoguang, Guangzhou Medical College, Guangzhou, China; Dr Mutya Macuno, Gov C Gallares Memorial Hospital, Bohol, the Philippines; Dr Jacinto BV Mantaring III, University of the Philippines, Manila, the Philippines, Dr Sujeewa Amarasena, Galle University, Galle, Sri Lanka; Dr Dayananda Bandara, Sri Lanka; Dr Prapaisri Layangool, Bhumibol Adulyadej Hospital, Bangkok, Thailand; Dr Pracha Nuntnarumit, Ramathibodi Hospital, Bangkok, Thailand; Dr Arvind Shenoi, Manipal Hospital, Bangalore, India; Dr Kin-Man Lui, Hospital C Conde S Januario, Macau; Dr Batsuvd Dashzeveg, Maternal and Child Medical Research Centre, Ulaanbaatar, Mongolia.

Competing interests: none declared

\section{REFERENCES}

1 de Man $\mathbf{P}$, Verhoeven BA, Verbrugh HA, et al. An antibiotic policy to prevent emergence of resistant bacilli. Lancet 2000;355:973-8.

2 Goossens H, Ferech M, Stichele RV, et al. Outpatient antibiotic use in Europe and association with resistance: a cross-national database study. Lancet 2005;365:579-87.

3 Turnidge J, Christiansen K. Antibiotic use and resistance: proving the obvious. Lancet 2005;365:548-9.

4 Gordon A, Isaacs D. Late onset infection and the role of antibiotic prescribing policies. Curr Opin Infect Dis 2004;17:231-6.

5 Stone PW, Gupta A, Loughrey M, et al. Attributable cost and length of stay of an extended-spectrum beta-lactamase-producing Klebsiella pneumoniae outbreak in a neonatal intensive care unit. Infect Control Hosp Epidemiol 2003;24:601-6

6 Lautenbach E, Patel J, Bilker W, et al. Extended spectrum beta lactamase producing Escherichia coli and Klebsiella pneumoniae. Risk factors for infection and impact of resistance on outcomes. Clin Infect Dis 2001;32:1162-71.

7 Gupta A. Hospital acquired infections in the neonatal unit: Klebsiella pneumoniae. Semin Perinatol 2002;27:340-5.

8 Royle J, Halasz S, Eagles G, et al. Outbreak of extended spectrum beta lactamase producing Klebsiella pneumoniae in a neonatal unit. Arch Dis Child Fetal Neonatal Ed 1999;80:F64-8

9 Pessoa-Silva CL, Meurer Moreira B, Almeida VC, et al. Extended-spectrum beta-lactamase-producing Klebsiella pneumoniae in a neonatal unit: risk factors for infection and colonisation. J Hosp Infect 2003;53:198-206.

10 Jain A, Indranil R, Mahendra K, et al. Prevalence of extended spectrum beta lactamase producing gram negative bacteria in septicaemic neonates in a tertiary care hospital. J Med Microbiol 2003;52:421-5.

11 Tullus K, Berglund B, Burman LG. Emergence of cross-resistance to betalactam antibiotics in fecal Escherichia coli and Klebsiella strains from neonates treated with ampicillin or cefuroxime. Antimicrob Agents Chemother, 1990;34, 361-2.

12 Paterson DL. Serious infections caused by enteric gram-negative bacilli: mechanisms of antibiotic resistance and implications for therapy of gramnegative sepsis in the transplanted patient. Semin Respir Infect 2002; 17:260-4.

13 Ariffin H, Navaratnam P, Kee TK, et al. Antibiotic resistance patterns in nosocomial gram-negative bacterial infections in units with heavy antibiotic usage. J Trop Pediatr 2004;50:26-31.

14 Isaacs D. Neonatal sepsis: the antibiotic crisis. Indian Pediatr 2005;42:1-5.

15 Vergnano S, Sharland M, Kazembe P, et al. Neonatal sepsis: an international perspective. Arch Dis Child Fetal Neonatal Ed 2005;90:F220-4.

16 Zaidi AKM, Huskins WC, Thaver D, et al. Hospital-acquired neonatal infections in developing countries. Lancet 2005;365:1175-88.

17 Karlowicz MG, Buescher ES, Surka AE. Fulminant late onset sepsis in a neonatal intensive care unit, 1988-1997, and the impact of avoiding empiric vancomycin therapy. Pediatrics 2000;107:1387-90.

18 Carling P, Fung T, Killion A, et al. Favorable impact of a multidisciplinary antibiotic management program conducted during 7 years. Infect Control Hosp Epidemiol 2003;24:699-706

19 Tiley SM, Macdonald JJ, Doherty PL, et al. Active promotion of antibiotic guidelines: an intensive program. Commun Dis Intell 2003;27(suppl):S13-18.

20 Gould IM. A review of the role of antibiotic policies in the control of antibiotic resistance. J Antimicrob Chemother 1999;43:459-65.

21 Isaacs D. Rationing antibiotic use in the neonatal unit. Arch Dis Child Fetal Neonatal Ed 2000;82:F1-2.

22 Isaacs D, Wilkinson AR, Moxon ER. Surveillance of colonisation and late-onset septicaemia in neonates. J Hosp Infect 1987; 10:114-19.

23 Gruson D, Hilbert G, Vargas F, et al. Strategy of antibiotic rotation: long-term effect on incidence and susceptibilities of Gram-negative bacteria responsible for ventilator-associated pneumonia. Crit Care Med 2003:31:1908-14.

24 Toltzis P, Dul MJ, lloyen C, et al. The effect of antibiotic rotation on colonization with antibiotic-resistant bacilli in a neonatal intensive care unit. Pediatrics 2002;110:707-11

25 Calil R, Marba ST, Von Nowakonski A, et al. Reduction in colonization and nosocomial infection by multiresistant bacteria in a neonatal unit after institution of educational measures and restriction in the use of cephalosporins. Am J Infect Control 2001;29:133-8.

26 Dietzman DE, Fischer GW, Schoenknecht FD. Neonatal Escherichia coli septicemia: bacterial counts in blood. J. Pediatr 1974;85:128-30.

27 Buttery JP. Blood cultures in newborns and children: optimising an everyday test. Arch Dis Child Fetal Neonatal Ed 2002;87:F25-8.

28 Solorzano-Santos F, Miranda-Novales MG, Leanos-Miranda B, et al. A blood microculture system for the diagnosis of bacteremia in pediatric patients. Scand J Infect Dis 1999:30:481-3.

29 Isaacs D, Wilkinson AR, Moxon ER. Duration of antibiotic courses for neonates. Arch Dis Child 1987;62:727-8.

30 Kaiser J, Cassat J, Lewno M. Should antibiotics be discontinued at 48 hours for negative late onset sepsis evaluations in the neonatal intensive care unit? J Perinatol 2002;22:445-7.

31 Isaacs D, Moxon ER. Handbook of neonatal infections. A practical guide. London: WB Saunders, 1999:57-60.

32 Webber S, Lindsell D, Wilkinson AR, et al. Neonatal pneumonia. Arch Dis Child 1990;65:207-11.

33 Goldmann DA, Weinstein RA, Wenzel RP, et al. Strategies to prevent and control the emergence and spread of antimicrobial resistant microorganisms in hospitals. A challenge to hospital leadership. JAMA 1996;275:234-40.

34 Adams-Chapman I, Stoll B. Prevention of nosocomial infections in the neonatal intensive care unit. Curr Opin Pediatr 2002;14:157-64. 